\title{
The Electronic Primary Care Research Network (ePCRN): A New Era in Practice-based Research
}

\author{
Kevin A. Peterson, MD, MPH, Patricia Fontaine, MD, and Stuart Speedie, PhD
}

The electronic Primary Care Research Network (ePCRN) is an electronic infrastructure that facilitates the conduct of randomized controlled trials (RCTs) in primary care and promotes the translation of research findings into practice. It provides a highly secure, Internet-based electronic infrastructure that will enable primary care practices anywhere in the United States to link with researchers in academic centers or the National Institutes of Health (NIH) to facilitate recruitment, entry, and follow-up of participants in multidisciplinary RCTs. The ePCRN also establishes a standardized clinic-based registry using distributed database technology to promote the translation of research findings into practice and to facilitate the process of clinical trials recruitment. The overall goals of the ePCRN are to provide the ability to perform large national collaborative studies throughout the United States, improve efficiency and reduce costs for individual trials, provide easier access for data retrieval and analysis, and involve primary care practices in the discovery and the translation of research findings into practice. ( $\mathrm{J}$ Am Board Fam Med 2006;19:93-7.)

More people in the United States visit a primary care provider over the course of a year than visit all other physicians combined. In 2001 , over $51 \%$ of all physician visits were to Family Medicine/General Practice, General Internal Medicine, or Pediatrics. ${ }^{1}$ Nevertheless, over the last 50 years the great majority of research funded by the National Institutes of Health $(\mathrm{NIH})$ has taken place in academic centers where less than $1 \%$ of patients are seen. $^{2}$ Historically, office-based primary care physicians have been isolated from the NIH research enterprise, and it has been estimated to take up to 17 years for $30 \%$ of evidence-based recommendations to be incorporated into medical care. ${ }^{3}$ The $\mathrm{NIH}$ has recognized that in order for the existing clinical research enterprise in the United States to remain successful, new partnerships with primary

Submitted 14 July 2005; revised 17 October 2005; accepted 21 October 2005.

From the Department of Family Medicine and Community Health (KAP, PF), and Division of Health Informatics (SS), University of Minnesota, Minneapolis, MN 55414.

This article is based on a presentation made at the 2005 Convocation of Practices, hosted by the American Academy of Family Physicians National Research Network and the Federation of Practice-based Research Networks, Colorado Springs, CO, March 2005.

Conflict of interest: none declared.

Corresponding author: Kevin A. Peterson, MD, MPH, Department of Family Medicine and Community Health, University of Minnesota, 925 Delaware Street, SE, Suite 220, Dinnaken Office Building, Minneapolis, MN 55414 (Email: peter223@umn.edu). care providers must be developed. ${ }^{4}$ Such partnerships should enhance the ability of clinical investigators to conduct research in their practices, as well as facilitate the rapid integration of new research findings into clinical care.

The electronic Primary Care Research Network (ePCRN) was funded in 2004 as part of the NIH Roadmap Initiative "re-engineering the clinical research enterprise" with the aim of addressing these issues. The purpose of this article is to describe the ePCRN and its potential to facilitate randomized controlled trials (RCTs) in primary care practices throughout the United States. The ePCRN will also provide an infrastructure that can present new research findings and evidence-based guidelines to clinicians in the context of a specific patient, leading to valuable applications for clinical performance monitoring and quality improvement efforts.

\section{Goals of the ePCRN}

The ePCRN integrates the practice-based research expertise from the community practices of the Federation of Practice-based Research Networks (FP$B R N$ ) with the advanced "Grid" electronic infrastructure of the University of Minnesota (UM), and the Midlands Research Practices Consortium (Mi$\mathrm{dReC}$ ), one of the largest PCRNs in the United Kingdom.

The specific goals of the ePCRN are to: 
1. Provide a highly secure, Internet-based electronic infrastructure that will enable primary care clinicians to work with researchers in academic centers or the NIH through a "web portal" for conducting RCTs.

2. Establish a standardized clinic-based patient registry that can be used by physicians in primary care practices to identify patients who are potentially eligible for these RCTs. The registry will also promote translation of research findings into clinical care by providing access to evidence-based guidelines in the form of reminders or prompts linked to individual patients' health records.

3. Develop a combined system using distributed database technology that interfaces with Open Grid Services Architecture (OGSA) and Web Services Resource Framework (WSRF). The combined system will allow real-time identification of potential. subjects, recruitment, online consent, and follow-up in RCTs. Benefits to clinicians will include a database that comprises all patients in the practice, suitable for analysis and tracking on the local level. Other potential benefits include video conferencing capability, support for clinical decision-making, and access to aggregate community performance benchmarks.

By achieving these goals, the ePCRN will provide the ability to perform large national collaborative studies with improved efficiency, reduced costs, and easier access for data retrieval and analysis. Primary care will be involved in the creation of new knowledge and its translation into practice, an important goal for the future of family medicine.

\section{Research Challenges Addressed by the ePCRN}

Randomized controlled trials form the foundation of systematic, evidence-based analysis. Conclusions drawn from RCTs are more likely to be appropriate and generalizable when the interventions are tested in the settings in which they will be applied. Although potentially rich sources of patients and data, primary care practices have not traditionally been sites for RCTs. Particular challenges for clinical trials in the primary care setting have included:

- Identifying and recruiting potential subjects for trials in a standardized way across many sites. ${ }^{5}$
- Clustering subjects by physician or by practice, which complicates analysis and compromises generalizability. ${ }^{6}$

- Recalling trial information when eligible patients arrive at a primary care office.

- Delivering complex interventions in a standard way across multiple sites.

- Tracking subjects as they change address or change practices during the trial follow-up.

- Privacy, confidentiality, and human subjects protection issues, because HIPAA and privacy standards restrict research access to private health information (PHI).

- The high cost of training and monitoring across a large geographic area, probably to rise still further with greater emphasis on privacy and confidentiality measures.

In parallel, in recent years there have been increased pressures on academic centers, the traditional site of the RCT. The growth of managed care has forced academic centers to compete with community hospitals and for-profit clinics for patients, whereas the emergence of proprietary contract-research organizations has siphoned off research funding. ${ }^{7}$

New partnerships between primary care practices and academic centers could be mutually beneficial in overcoming these difficulties, and the ePCRN applies emerging technologies toward that goal. In particular, the introduction of very highspeed backbone networking supports the integration of large numbers of primary care physicians and their practice populations into large multisite clinical trials.

\section{ePCRN Infrastructure}

The electronic infrastructure of the ePCRN is being built on a web-enabled distributed database technology that makes use of cutting-edge web technologies such as the OGSA and WSRF. This allows creation of distributed clinical information systems located at the site of practice that can be appropriately and securely linked together. Exciting possibilities for this technology include the potential for patient eligibility searches across wide geographic areas, real-time video conferencing, implementation of medical record communication standards, and real-time clinician access to standard clinical performance measures. This ePCRN infra- 
structure will help to form the foundation for a revolution in clinical research in primary care in the United States.

The ePCRN depends on "Internet2," which was created in October of 1994, when 34 universities in the United States announced plans to build a faster, more powerful network for academic purposes. This development was stimulated by a shift of the National Science Foundation from the government-subsidized national backbone (NSFNET) to the privatized Internet. By that time the capacity of the existing Internet was already being strained by an enormous growth in use. Because of the increased use of multimedia, the pressure on the existing Internet was expected to continue to increase, and the limited availability of bandwidth was not sufficient to meet scientific and academic demands. In addition to upgrading equipment and protocols, Internet 2 connected participants and institutions in planned, end-to-end connectivity using direct transmission routes implemented through high-capacity "points of presence" termed "GigaPops." GigaPops connect urban area networks, state and regional networks, university campuses, and labs, all at speeds up to 10 Gigabits per second (Gbps), compared with 45 megabits per second (Mbps) on the existing Internet backbone.

The backbone of Internet2 is known as "Abilene." Abilene provides very high-speed backbone network service (VBNS) capability to many locations across the country. GigaPops then distribute this rapid communication potential within regions. The current partners in the development of the ePCRN, the UMN and the UCSF, were among the founding members of the Abilene network. They will use their pioneering expertise to advance the current medical infrastructure by combining aspects of the Internet and Internet2 into a system with substantially greater potential than its predecessors.

\section{Current Status of the ePCRN}

The ePCRN infrastructure is being introduced through the Federation of PBRNs (FPBRN). Currently composed of 53 regional and national PBRNs with more than 6500 physicians performing research in $>2700$ primary care practices, the FPBRN serves a patient population of approximately 16 million people.

Ten PBRNs are currently registering primary care physicians (PCPs) and research associates who have expressed interest in participating in the ePCRN (see ePCRN Core Networks in Acknowledgments). In late 2005, more than 100 PCPs will participate in the first RCT, which will test the ePCRN's technical capabilities and evaluate optimal procedures for data management.

Specific steps in the development of the ePCRN have occurred as follows:

\section{Step 1: Development of the Web Portal for Clinical Trials}

Figure 1 demonstrates the basic schematic for the web portal.

The ePCRN staff has worked with researchers from MidReC (Birmingham, England) to adapt and upgrade the technologic infrastructure that supports clinical trials in primary care clinics throughout the United Kingdom. Created initially at the University of Minnesota, this class 5 government security rated tool provides a web portal to a collection of databases, management and analysis tools, and research resources. Access to the site is obtained by PBRN members through their participating PBRN director, following documentation of human subjects training and completion of basic security requirements. An RSA security fob, a small electronic device the size of a car remote, serves as the "key" into the system, allowing participating PCPs highly secure access to the ePCRN from any computer terminal.

As clinical trials are introduced, primary care practices clinic will be able to use applications designed either locally at the PBRN, or centrally by a clinical research group. These applications will assist in the identification, randomization, and follow up of subjects entered into a specific clinical trial by the practice. Entering a subject in a clinical trial will result in appropriate compensation to the practice and to the participating PBRN. This avoids the persistent problem tolerated too long by primary care physicians, namely, participating in research at the cost of losing revenue from clinical activity. By providing a new revenue stream and insuring that time spent will be reimbursed at reasonable rates, ePCRN research activity becomes a feasible and attractive prospect for PCPs.

\section{Step 2: Creation of a standardized patient registry}

To provide support for patient recruitment and for potential clinic translation efforts, a standardized patient registry will be created at participating clin- 


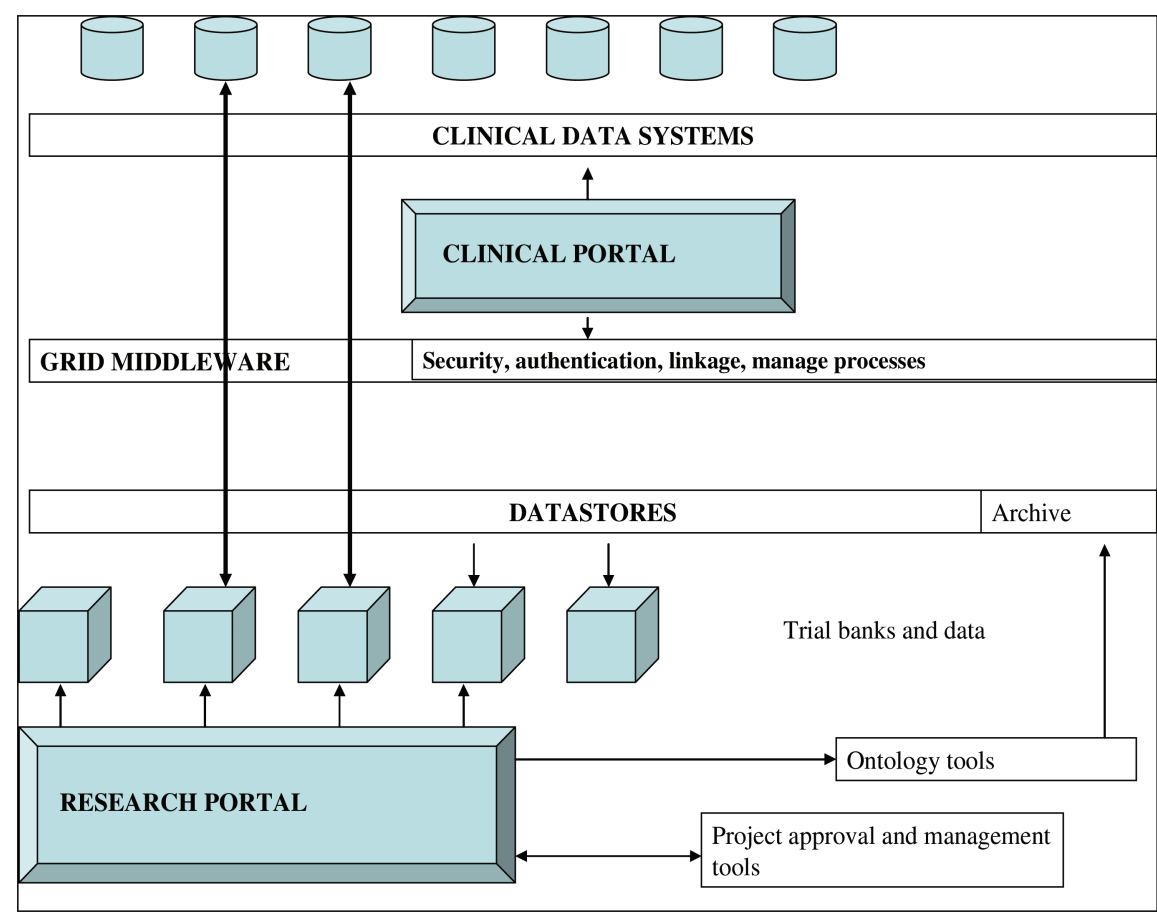

Figure 1. Web portal schematic

ics. This registry will exist as a data warehouse with standardized data capture and delivery capabilities. Initially the data warehouse will be built from downloads from the patient billing and laboratory information systems at 100 primary care clinics. As the project proceeds, OGSA web services will allow development of distributed databases (ie, databases in multiple locations) that provide sophisticated security and controlled access. By instituting a standard electronic interface, the data warehouse can be used to transfer data between electronic health records (EHRs).

The creation of a standardized patient registry within a clinic provides substantial additional functionality. Because the data set will contain ICD-9 and CPT codes, it will act as a single disease registry, eliminating the current pressure to create a separate disease registry for each medical condition a practice wishes to track or evaluate. Practices can use their registries for quality improvement activities, and provide the PCP with a resource for examining aggregate clinical measures. The registry can also support a number of "translation tools," such as patient-specific prompts for testing and treatment, which serve to link practicing physicians with evidence-based guidelines at the point of care. For example, the registry can be set up with prompts to let a physician know when an individual diabetic patient's glycosylated hemoglobin value is not within target range or when an asthmatic patient needs an increase in stepped care.

The standardized registry differs substantially from the EHR itself. The user interface of the registry is very strictly defined, as opposed to the more flexible interface required by an EHR. The EHR focuses on individual history across time, whereas the registry is built to examine aggregated patient groups by disease. The registry facilitates population-based audit and feedback whereas the EHR optimizes storage, billing, and clinical activity. Finally, the registry will provide an interface with clinical research activities within the primary care environment.

The ePCRN is currently working with the AAFP to integrate the standardized registry and 18 separate EHR vendors through the American Society of Technology and Measurement medical communication standard known as the Continuity of Care Record. It is expected that this communication standard will play an important role in communications in primary care, and should be identified as a desirable and potentially important characteristic when evaluating the introduction of an EHR in a primary care setting. 


\section{Future Directions and Opportunities}

The role of emerging Grid technologies is to provide seamless and scalable access to wide-area distributed resources, to enable the sharing, scheduling and aggregation of a wide variety of geographically distributed computational and data resources and present them as a single, unified resource - a virtual laboratory or organizationaccessible from the desktop. Primary care practitioners are distributed geographically, and are often isolated from academic centers. Scaled to the size of the population and viewed as a network of patient records stored at individual practices, the primary care infrastructure represents a large, complex, and heterogeneous dataset in which data are stored as a "federation" of databases. During the second and third years of the ePCRN's NIH Roadmap Initiative funding, we intend to develop open source Web services that will support and build on the translation/patient registry and RCT platforms. Enhanced functionality will include prompts for PCPs in participating practices when a patient is eligible to participate in a particular clinical trial. Prompts will be linked to patients' EHRs without any private health information being passed outside the primary care clinic. This provides a simple and automatic system for patient recruitment that minimizes disruption in the PCP's schedule, and leaves the choice of participation in a clinical trial up to the patient and the PCP.

The research portal will also provide analytic tools for researchers to examine their clinical trial data in real time. Accessibility of clinical trial data for the public will be promoted by developing an integrated solution with the trial bank at UC San Francisco. By integrating standard terminology developed in cooperation with the National Cancer Institute, ePCRN datasets will provide for better meta-analysis, and allow development of sophisticated clinical trial development tools that simplify both planning and implementation of practicebased research. Finally, live interactive video conferencing through open source Access Grid Nodes will be available for clinics having access to satisfactory bandwidth. This will allow for ease and flexibility with training, preparation, and communication issues both for central research organizations and for clinical researchers.

\section{Conclusions}

The Future of Family Medicine project ${ }^{8}$ has identified critical new directions that will be necessary for primary care to succeed as we move into the future. Recommendation number 5, on Enhancing the Science of Family Medicine, states:

"Participation in the generation of new knowledge will be integral to the activities of all family physicians and will be incorporated into family medicine training. Practice-based research will be integrated into the values, structures, and processes of family medicine practices."

Technologic and methodological advances now hold promise to integrate research activities into primary care without the cost and compromises previously entailed. Development of the ePCRN and participation from PBRNs throughout the country will enhance our ability to contribute to new knowledge and to improve patient care.

\section{References}

1. Cherry DK, Woodwell DA. National Ambulatory Medical Care Survey: 2000 summary. Adv Data 2002;328: 1-32.

2. Green LA, Fryer GE Jr, Yawn BP, Lanier D, Dovey SM. Ecology of medical care. N Engl J Med 2001; 344:2021-5.

3. Committee on Quality of Health Care in America, Institute of Medicine. Crossing the quality chasm: a new health system for the 21st century. Washington (DC): National Academy Press; 2001

4. Re-engineering the clinical research enterprise. 2005 [cited $2005 \mathrm{Jul}$ 8]. Available from http://nihroadmap.nih.gov/clinicalresearch/index.asp.

5. Foy R, Parry J, Duggan A, et al. How evidence-based are recruitment strategies for randomized controlled trials in primary care? Experience from seven studies. Fam Pract 2003;20:83-92.

6. Croughan M. Generalizability in PBRN studies. In: Methods for practice-based research networks: challenges and opportunities. Proceedings from the Practice-Based Research Network Methods Conference 2001; Leawood (KS): American Academy of Family Physicians; 2001 December.

7. Blumenstyk G, Wheeler DL. Academic medical centers race to compete in the \$3.2-billion drug-testing market. Chronicle of Higher Education [accessed 20 Mar 1998]. Available from http://chronicle.com/ che-data/articles.dir/art-44.dir/issue-28.dir/ 28a00101.htm.

8. Future of Family Medicine Project Leadership Committee. The future of family medicine: a collaborative project of the family medicine community. Ann Fam Med 2004;2:S3-32. 